\title{
Turbulent Stresses in Local Simulations of Radiation-Dominated Accretion Disks, and the Possibility of the Lightman-Eardley Instability
}

\author{
Shigenobu Hirose \\ Institute for Research on Earth Evolution, JAMSTEC, Yokohama, Kanagawa 236-0001, \\ Japan \\ Omer Blaes \\ Department of Physics, University of California, Santa Barbara, Santa Barbara CA 93106 \\ and \\ Julian H. Krolik \\ Department of Physics and Astronomy, Johns Hopkins University, Baltimore, MD 21218
}

\begin{abstract}
We present the results of a series of radiation-MHD simulations of a local patch of an accretion disk, with fixed vertical gravity profile but with different surface mass densities and a broad range of radiation to gas pressure ratios. Each simulation achieves a thermal equilibrium that lasts for many cooling times. After averaging over times long compared to a cooling time, we find that the vertically integrated stress is approximately proportional to the vertically-averaged total thermal (gas plus radiation) pressure. We map out-for the first time on the basis of explicit physics - the thermal equilibrium relation between stress and surface density: the stress decreases (increases) with increasing surface mass density when the simulation is radiation (gas) pressure dominated. The dependence of stress on surface mass density in the radiation pressure dominated regime suggests the possibility of a Lightman-Eardley inflow instability, but global simulations or shearing box simulations with much wider radial boxes will be necessary to confirm this and determine its nonlinear behavior.
\end{abstract}

Subject headings: accretion, accretion disks — instabilities - MHD - X-rays: binaries 


\section{Introduction}

It has long been known that models of optically thick, geometrically thin accretion disks based on the alpha stress prescription of Shakura \& Sunvaev (1973) are subject to thermal and inflow ("viscous") instabilities when the vertically-averaged radiation to gas pressure ratio exceeds 3/2 (Lightman \& Eardley 1974; Shibazaki \& Hōshi 1975; Shakura \& Sunyaev 1976). Such radiation pressure dominated accretion disks are expected to be relevant for luminous active galactic nuclei and quasars as well as for thermal states of X-ray binaries. However, with one possible exception, there has never been clear observational evidence, or even observational motivation, for the existence of these instabilities in these sources. This is in marked contrast to the cases of dwarf novae and soft X-ray transients, where thermal instabilities in the disk associated with hydrogen ionization, not radiation pressure, are central to explaining the observed outbursts (Lasota|2001). The X-ray binary GRS 1915+105 does exhibit recurrent outburst behavior that has been modelled as being due to radiation pressure driven instabilities (Belloni et al. 1997), but it is far from clear that this is the correct explanation. This source is the brightest among Galactic black hole X-ray binaries, and spends considerable time at super-Eddington luminosities (Done, Wardziński, \& Gierliński 2004). Other black hole X-ray binaries commonly reach high enough Eddington ratios for instabilities to exist according to standard accretion disk theory, but do not exhibit similar variability.

It is now widely suspected that accretion stresses in black hole accretion disks are due to turbulence related to the nonlinear growth of the magnetorotational instability (MRI) (Balbus \& Hawley 1998). It is computationally feasible to perform thermodynamically consistent, radiation MHD simulations of this turbulence in local patches of accretion disks. These stratified shearing box simulations fully capture grid-scale numerical losses of energy as heat and also account for radiative heat losses within the flux-limited diffusion approximation (Hirose, Krolik, \& Stone 2006). Such simulations have now been performed for a broad range of radiation to gas pressure ratios, and in each case an approximate thermal equilibrium has been established lasting for many cooling times (Hirose. Krolik, \& Stone 2006; Krolik, Hirose, \& Blaes 2007; Hirose, Krolik, \& Blaes 2009). No sign of the radiation pressure thermal instability is present, even at radiation to gas pressure ratios well above the instability threshold of the standard alpha model (Hirose, Krolik, \& Blaes 2009; see also Turner 2004).

Thermal stability exists because the stress-pressure relation assumed by the standard alpha-model is only established on time scales longer than the thermal time 1 The causal

\footnotetext{
${ }^{1}$ In a prescient comment, Lightman \& Eardley (1974) suggested that the alpha prescription might only
} 
direction of the relation is from stress to pressure, not from pressure to stress. Turbulence is chaotic and results in a highly fluctuating dissipation rate. It is that dissipation that ultimately changes the pressure, but that pressure response is only established after a thermal time. An upward fluctuation in pressure does not result in an upward stress response on this time scale as the causal direction is the other way round. Hence there is no positive feedback loop on the thermal time scale that would result in a thermal runaway (Hirose, Krolik, \& Blaes 2009).

This still leaves open the question of the slower inflow instability. Mass and angular momentum conservation imply that radial mass transport in an accretion disk with a local turbulent stress is governed by the equation Lightman \& Eardley 1974; Lynden-Bell \& Pringle 1974)

$$
\frac{\partial \Sigma}{\partial t}=\frac{1}{r} \frac{\partial}{\partial r}\left[\frac{1}{\ell^{\prime}} \frac{\partial}{\partial r}\left(r^{2} W_{r \phi}\right)\right],
$$

where $\Sigma$ is the local surface mass density, $r$ is the radius, $\ell^{\prime} \equiv d \ell / d r$ is the radial derivative of specific angular momentum, and $W_{r \phi}$ is the vertically integrated turbulent stress. For geometrically thin disks, the inflow time is much longer than the thermal time, so both thermal and vertical hydrostatic equilibrium should be maintained over the time scales associated with mass transport. Assuming the disk is optically thick and cools through radiative losses (which is the case for all of our stratified shearing box simulations so far; Hirose, Krolik, \& Stone 2006; Krolik, Hirose, \& Blaes 2007; Hirose, Krolik, \& Blaes 2009), then radiative, hydrostatic, and thermal equilibrium imply that the vertically-averaged stress in a radiation pressure dominated disk is simply given by

$$
\tau_{r \phi}=\frac{c \Omega^{2}}{\kappa r\left|\Omega^{\prime}\right|}
$$

where $\Omega$ is the angular velocity in the disk, $c$ is the speed of light, and $\Omega^{\prime} \equiv d \Omega / d r$ is the shear (Shakura \& Sunyaev 1976). The opacity $\kappa$ is generally dominated by electron scattering in this regime, and is therefore constant. Hydrostatic equilibrium implies that the disk half-thickness is $H \sim 2 P /\left(\Omega^{2} \Sigma\right)$, where $P$ is the midplane pressure. Hence at a particular radiation pressure dominated radius,

$$
W_{r \phi} \sim 2 H \tau_{r \phi} \propto \frac{P}{\Sigma} .
$$

A standard alpha disk model with $\tau_{r \phi}=\alpha P$ implies from equation (2) that $P$ is independent of $\Sigma$, so $\partial\left(W_{r \phi}\right) / \partial \Sigma<0$. Equation (1) therefore represents a diffusion equation with a negative diffusion coefficient. Unstable growth of surface density enhancements and rarefactions would therefore result (Lightman \& Eardley 1974; Lightman 1974a, b).

be valid on slow time scales, which they identified as being of order the inflow time and longer. 
Like the fictitious thermal instability, however, the reality of the inflow instability has always been questionable. For example, as pointed out by Lightman \& Eardley (1974) themselves, a stress proportional to the gas pressure alone would produce an inflow stable disk. On the other hand, the stratified shearing box simulations appear to be consistent with total thermal pressure scaling with stress on supra-thermal time scales (Hirose, Krolik, \& Blaes 2009), a fact that we will demonstrate much more explicitly in this paper.

A plot of vertically integrated stress $W_{r \phi}$ versus surface density for a range of thermal equilibria at a fixed radius within a disk would suggest inflow stability or instability depending on whether the slope is positive or negative, respectively. Because thermal equilibrium implies that the local radiation flux $F$ emerging from each face of the disk is given by $F=W_{r \phi} r\left|\Omega^{\prime}\right| / 2$, a plot of $F$ (or effective temperature) versus surface density may be used in the same way. Such "S-curve" plots are commonly used to investigate the hydrogen ionization-driven disk instabilities in dwarf novae and soft X-ray transients (e.g. Smak 1984; Lasota 2001).

Using radiation MHD simulations of stratified shearing boxes, we have now mapped out the stress versus surface density thermal equilibrium curve for a wide range of radiation to gas pressure ratios at a fixed radius in a disk around a stellar mass black hole. This is the first time that this curve has been drawn on the basis of explicit physical mechanisms, rather than phenomenological estimates. While there are large fluctuations which produce an inherent scatter in the thermal equilibrium curve, the results are consistent with the standard alpha disk model. The inflow instability might therefore be present even in MRI turbulent disks.

This paper is organized as follows. In section 2 we provide a brief overview of the numerical parameters and properties of the simulations. In section 3 we discuss the stresspressure relation and demonstrate that average total thermal pressure, rather than a pressure which singles out gas pressure as being special in some way, is best correlated with average stress. In section 4, we summarize the resulting thermal equilibria on a stress-surface density diagram, and discuss the possible implications. We summarize our conclusions in section 5 .

\section{Simulations}

The radiation MHD equations for stratified shearing boxes, and the numerical methods we use to solve them, have been described in detail by Hirose, Krolik, \& Stone (2006) and Hirose, Krolik, \& Blaes (2009), and references therein. Grid scale losses of mechanical and magnetic energy are fully captured as heat in the gas, and gas and radiation exchange heat 
through Planck mean free-free absorption and emission and Compton scattering. Radiation transport is treated through flux-limited diffusion.

All the simulations were run with an angular velocity $\Omega=190 \mathrm{~s}^{-1}$, corresponding to a radius of $30 G M / c^{2}$ around a $6.62 \mathrm{M}_{\odot}$ Schwarzschild black hole. Different total surface mass densities were chosen for each simulation in order to map out the stress-surface density relation. Table 1 summarizes the parameters of each simulation. The $x, y$, and $z$ axes correspond to the radial, azimuthal, and vertical directions, respectively. The simulations were initialized in hydrostatic and thermal equilibrium under an assumed initial vertical profile of dissipation per unit volume proportional to the density divided by the square root of the optical depth measured from the nearest surface. Once the simulation starts, the dissipation is thereafter self-consistently determined from the turbulent dynamics. Each simulation started with a weak magnetic field consisting of a twisted azimuthal flux tube located at the center of the box. We refer the reader to Hirose, Krolik, \& Blaes (2009) for more details.

The vertical box height $L_{z}$ of the simulations was chosen so that two conditions would be satisfied (Hirose, Krolik, \& Blaes 2009). First, the total surface mass density changes by less than a few percent due to vertical mass loss and mass creation by the density floor of the simulation. Second, the MRI is always well-resolved in the midplane regions. These conditions were checked a posteriori. The upper and lower photospheres are always within the simulation domain, although the optically thin regions do not necessarily have a tremendous vertical extent. This should not significantly affect the emergent flux, which is what really matters. Because the radiation energy density in the optically thin regions is nearly independent of height, the radiation energy density at the photospheres should depend only weakly on box height. It is this radiation energy density that provides the effective boundary condition for the radiation diffusion equation in the optically thick regions where the vast majority of the dissipation occurs, so the emergent flux should be well-determined. Nevertheless, we warn the reader that we have not demonstrated numerical convergence with respect to variations in the simulation box dimensions.

Six of the simulations (0211b, 0519b, 1112a, 1126b, 0520a, and 0320a) were initialized as radiation pressure dominated, while two (090304a and 090423a) started out gas pressure dominated. (Due to an initialization error, simulations 090304a and 090423a actually had an angular velocity $1.6 \%$ smaller than the others: $187 \mathrm{rad} \mathrm{s}^{-1}$ rather than $190 \mathrm{rad} \mathrm{s}^{-1}$. We do not believe this significantly affects our conclusions, as the fluctuations in stress and radiative cooling are considerably larger than this.) The results of simulations $1112 \mathrm{a}$ and 1126b were discussed extensively in Hirose, Krolik, \& Blaes (2009).

All of the simulations share many of the properties that we discussed in detail in previous 
papers on radiation MHD simulations of stratified shearing boxes (Hirose. Krolik. \& Stone 2006; Krolik, Hirose, \& Blaes 2007; Blaes, Hirose, \& Krolik 2007; Hirose, Krolik, \& Blaes 2009). The subphotospheric regions consist of a magnetorotational turbulent zone in the midplane regions which is supported against gravity by gas and radiation pressure gradients. Further out, magnetic forces dominate or contribute substantially, and Parker instability dynamics, rather than MRI turbulence, appear to control the structure of the outer layers.

Figure 1 shows the thermal and turbulent energy content in the box as a function of time for each of the radiation pressure dominated simulations. The thermal history of the more gas-dominated simulations are shown in Figure 2, Defining the instantaneous thermal time as the total radiation and gas internal energy divided by the emergent radiative flux on both vertical faces of the box, the thermal time averaged over the duration of each of the simulations ranges from a minimum of 6 orbits for 090423a to a maximum of 24 orbits for $0519 \mathrm{~b}$. All the simulations have reached an approximate thermal equilibrium, albeit with long time scale fluctuations. There is no evidence for the thermal instability predicted by classic alpha disk models (Shakura \& Sunvaev 1976), in spite of the fact that the timeaveraged ratio of vertically-averaged radiation pressure to vertically-averaged gas pressure is as high as 70 in the case of simulation $0519 \mathrm{~b}$.

\section{The Stress-Pressure Relation}

A number of authors have suggested alternative stress prescriptions in which the accretion stress is proportional to gas pressure or some combination of gas and radiation pressures, rather than total thermal pressure (gas plus radiation), in part to stabilize the radiation pressure dominated portion of black hole accretion disks (Sakimoto \& Coroniti 1981; Stella \& Rosner 1984; Burm 1985; Szuszkiewicz 1990; Merloni \& Fabian 2002; Merloni 2003). Sakimoto \& Coroniti (1989) have even argued that standard alpha disk models are inconsistent in the radiation pressure dominated regime, as magnetic fields that are strong enough to provide accretion stress would be too buoyant to be retained by the disk. Their argument has two flaws, however. First, they assumed that the magnetic field consisted of discrete flux tubes, rather than being more continuously distributed throughout the plasma. Second, they were unaware at the time of magnetic field generation by the MRI. Note that there is no indication in the energy histories shown in Figure 1 that the magnetic and turbulent kinetic energies are limited by the gas internal energy. Indeed, in the two most radiation pressure dominated simulations (0211b and 0519b), the magnetic energy almost always exceeds the gas internal energy, and even the turbulent kinetic energy can occasionally be larger than the gas internal energy. 
Based on the behavior of the stress to thermal pressure ratio within simulation 1112a, we argued in the past that a prescription where stress and total thermal pressure are proportional to each other (on time scales longer than the thermal time) is a superior description of the simulation behavior than alternatives where the pressure is taken to be just the gas pressure alone or the geometric mean of radiation and gas pressure (see Fig. 10 of Hirose, Krolik, \& Blaes 2009). A similar conclusion can be drawn when data is compared across the simulations we are considering here.

For each of our simulations, we computed the vertically-averaged stress $\tau_{r \phi, \text { av }}$ and the box-average of various measures of pressure $P_{\mathrm{av}}$ : the sum of the radiation pressure $P_{\mathrm{rad}}$ and gas pressure $P_{\text {gas }}$, the geometric mean $\left(P_{\text {rad }} P_{\text {gas }}\right)^{1 / 2}$ of these pressures, and just the gas pressure. We then computed the time average of the ratio of these spatial averages of stress and pressure, $\alpha \equiv<\tau_{r \phi, \mathrm{av}} / P_{\mathrm{av}}>$, and compared them to the time average of the ratio of spatially averaged radiation and gas pressure, $\left\langle P_{\text {rad,av }} / P_{\text {gas,av }}\right\rangle$. These time averages were computed over the entire duration of each simulation, excluding the first 10 orbits as the MRI was still in its growth phase.

The results are shown in Figure 3. The black points show the results for $P_{\text {av }}$ defined as the total pressure, and are clearly closest to being independent of the radiation to gas pressure ratio. (A linear fit to the dependence of these values of $\alpha$ on the radiation to gas pressure ratio gives a slightly negative slope which is consistent with zero within one standard deviation of the slope determination.) A weighted average of these values gives $\bar{\alpha}=0.018 \pm 0.002$. The green curve shows what one would obtain if stress and total pressure were proportional with this ratio, but we redefined $\alpha$ in terms of the geometric mean of radiation and gas pressure, i.e.

$$
\left\langle\frac{\tau_{r \phi, \mathrm{av}}}{P_{\mathrm{av}}}\right\rangle \simeq \bar{\alpha}\left[\left\langle\frac{P_{\mathrm{rad}, \mathrm{av}}}{P_{\text {gas }, \mathrm{av}}}\right\rangle^{1 / 2}+\left\langle\frac{P_{\mathrm{rad}, \mathrm{av}}}{P_{\mathrm{gas}, \mathrm{av}}}\right\rangle^{-1 / 2}\right] .
$$

The blue curve shows the same thing for the gas pressure stress prescription,

$$
\left\langle\frac{\tau_{r \phi, \mathrm{av}}}{P_{\mathrm{av}}}\right\rangle \simeq \bar{\alpha}\left(1+\left\langle\frac{P_{\mathrm{rad}, \mathrm{av}}}{P_{\mathrm{gas}, \mathrm{av}}}\right\rangle\right)
$$

These curves clearly explain the trends seen in the data for these alternative stress prescriptions.

Our results are therefore most consistent with the standard alpha prescription involving total thermal pressure. Recent axisymmetric global radiation MHD simulations by Ohsuga et al. (2009) also reach a similar conclusion. We emphasize, however, that such a prescription should still be treated with caution. There is no obvious reason that $\alpha$ should 
be a universal constant, and we do not know what physics is determining its value in our simulations. It is possible, for example, that $\alpha$ is a function of $\Omega$ or $M$, as we have not varied these parameters at all; global simulations Hawley \& Krolik (2001, 2002) have shown that it can be a function of radius when the underlying orbital dynamics change. In fact, it is rather surprising that it is as constant as it is when defined in terms of total thermal pressure. Moreover, as we emphasized in Hirose, Krolik, \& Blaes (2009), the fact that stress is in any way related to pressure is really because turbulent dissipation heats the plasma. Only when averaged over height and averaged over many thermal times does the standard alpha prescription provide an adequate description of the fact that pressure is correlated with stress.

\section{The Stress-Surface Density Relation}

For each simulation, we computed the vertically integrated stress as a function of time, and then time averaged this over the simulation's duration, again ignoring the first ten orbits. The results are plotted as a function of surface density in Figure 4. Vertical error bars indicate the standard deviations of the instantaneous fluctuations in stress about the mean. (Due to mass loss and an imposed density floor, the surface density also fluctuates, but by at most two percent in all the simulations.) The right hand axis indicates the effective temperature of the radiation leaving each vertical face of the box if perfect thermal equilibrium held. We have also computed versions of the diagram by time-averaging the radiation flux leaving both faces of the box and computing the effective temperature, and time-averaging the volumeintegrated dissipation rate. All three versions are almost identical, as they must be, given the approximate thermal equilibrium that has been established in each simulation.

The curves in Figure 4 show the results predicted by standard alpha disk models with stress scaling with total pressure, but with internal structure parameters based on the vertical structure observed in the simulations themselves. These parameters are defined in Appendix A, and listed in Table 2, The simulation data are clearly consistent with the alpha disk model, although there are variations in the internal structure parameters that, when averaged over all the simulations, produce an alpha disk curve that misses many of the data points (solid curve in Figure 4). Note that the alpha disk model predicts a maximum value of surface density $\Sigma_{\text {crit }}$ above which an alpha disk cannot be in thermal equilibrium. We have not attempted to simulate such high surface densities.

The negative slope of the stress-surface density relation in the radiation pressure dominated simulation data indicates a negative mass diffusion coefficient, suggesting inflow instability if local thermal equilibrium is everywhere maintained on the time scales associated with 
nonlocal, radial mass transport. Because this would be a diffusive instability, a perturbation with radial wavelength $\lambda$ would have a characteristic growth rate $\sim \alpha \Omega(H / \lambda)^{2}$ (Lightman 1974b; Shakura \& Sunyaev 1976), hence growing faster at shorter wavelengths. However, there must be a short wavelength cutoff to this trend. Lightman (1974b) argues that this cutoff is of order the vertical scale height $H$ because of turbulent mixing on such length scales. Shakura \& Sunyaev (1976) reach approximately the same conclusion based on the fact that the linear thermal and inflow unstable modes of the alpha disk equations become degenerate and then stable for radial wavelengths less than of order the disk scale height. This latter fact suggests that the short wavelength cutoff to the inflow instability might actually be considerably larger than the disk scale height, because the radiation pressure driven thermal instability itself does not manifest itself in real MRI turbulence. Insofar as thermal effects become increasingly important to the inflow instability at short wavelengths, this may make the cutoff wavelength longer.

Because the radial extent of our simulation domain is so small (significantly less than a scale height in all cases - see Table 1), we do not expect (and do not find) the inflow instability to be present in our simulations.2 In principle, vertically stratified shearing boxes with much wider radial domains could produce the instability, even though the shearing box has no net radial accretion. In spite of our use of the term "inflow" instability, the instability is really one of negative diffusion, and can grow even in the absence of a net mass accretion flow through the box. We can demonstrate this fact explicitly by vertically integrating the shearing box equations (see Appendix B). This results in a radial mass diffusion equation,

$$
\frac{\partial \Sigma}{\partial t}=\frac{1}{(2-q) \Omega} \frac{\partial^{2}}{\partial x^{2}} W_{x y},
$$

identical to the local limit of equation (1), where $q \equiv-d \ln \Omega / d \ln r$ and $|x| \ll r$. If $\partial W_{x y} / \partial \Sigma<0$ under conditions of thermal equilibrium, we would then expect inflow instability. Stratified shearing box simulations sufficiently wide in the radial direction might therefore manifest this instability in the radiation pressure dominated regime. To see this effect may require radial box widths much larger than the vertical pressure scale height, in

\footnotetext{
${ }^{2}$ Because the thermal instability of the alpha disk also has a short radial wavelength cutoff, the reader might wonder how we can claim with confidence that the thermal instability does not exist based only on shearing box simulations with narrow radial domains (Hirose, Krolik, \& Blaes 2009). This is because an infinite radial wavelength mode (one that does not vary at all in radius) can still be present in such boxes, and would still grow if it were truly thermally unstable. Physically, the pure thermal instability of the standard alpha disk arises because the vertically integrated heating rate is more temperature sensitive than the cooling rate, and radial variations are irrelevant. In contrast, the growth rate of infinite radial wavelength modes on the inflow instability branch is zero.
} 
order that different radial regions be thermally decoupled and that stress scale with local pressure on time scales longer than the local thermal time. In any case, such simulations would enable a determination of whether the inflow instability is real, and what its minimum and fastest growing wavelengths are.

If the instability does manifest itself in MRI turbulence, such simulations would also help determine its nonlinear outcome. Of course, a number of hydrodynamic simulations have been done of the combined thermal/inflow instability of radiation dominated alpha-disks (e.g. Honma, Matsumoto, \& Kato 1991; Szuszkiewicz 1998). However, the time evolution of these simulations is dominated by the faster thermal instability, which we now know to be fictitious. As far as we are aware, the only simulations that have ever been attempted of the radiation dominated inflow instability on its own were done by Lightman (1974b) himself, who numerically solved the alpha disk mass diffusion equation, assuming that thermal equilibrium is strictly maintained. All his simulations therefore started with a surface density less than $\Sigma_{\text {crit }}$. The resulting evolution rapidly produced clumping of the surface density up to $\Sigma_{\text {crit }}$ with optically thin rarefied regions in between. Both of these conditions violated the assumptions on which the mass diffusion equation is based, and the numerical calculation had to be stopped. As Lightman (1974a) pointed out, surface densities exceeding $\Sigma_{\text {crit }}$ cannot be in thermal equilibrium within the assumptions of the alpha model, because heat generation always exceeds cooling through vertical radiative diffusion. On the other hand, if the characteristic radial size of the clumps is as small as the disk scale height, radial heat transport is likely to be important in the nonlinear outcome. Radiation MHD simulations with radially wide shearing boxes could address all these issues.

Radiation MHD simulations could also clear up another question clouding prediction of the outcome of this putative instability: the negative slope of the stress-surface density relation on the radiation dominated branch implies growing surface density fluctuations only if local thermal equilibrium with purely vertical heat flow is maintained everywhere while the instability tries to develop. It could be that subtleties in the behavior of MRI turbulence preclude this from happening, just as the thermodynamics of the turbulence prevented the thermal instability from manifesting itself.

This issue is closely related to the question of exactly what the gas-dominated and radiation-dominated branches of equilibria in Figure 4 truly represent. If this were a lowdimensional dynamical system like the standard alpha-disk, then the fact that both branches are thermally stable would imply an unstable equilibrium branch in between. However, a stratified shearing box with real turbulence is not a low-dimensional dynamical system - the spatial dependence of gas density, pressure, velocity, magnetic field, and radiation pressure all influence its evolution, and Figure 4 is probably better viewed as a projection of a very 
complicated dynamical phase space. In our experience, simulations that are initialized far away from the equilibrium branches do not undergo steady heating or cooling, but instead wander chaotically due to the fluctuating character of the turbulence. Whether there is a true unstable thermal equilibrium branch between the gas and radiation-dominated branches would be very well masked by this stochasticity even if the vertically-integrated stress were the only significant dynamical variable; the much higher dimensionality of the real system makes it essentially impossible to test whether such a branch exists on the basis of simulation data. It is conceivable that a local perturbation in surface density would require considerable time to reach the thermal equilibrium branch, and would in any case fluctuate about that branch. It is an open question whether or not this evolution would be conducive to inflow instability.

It is also noteworthy that hydrostatic and radiative equilibrium necessarily enforce a characteristic stress on the radiation dominated branch (equation 2, Shakura \& Sunvaev 1976). There is no such constraint on the gas dominated branch, and in fact hydrostatic equilibrium is not even needed to derive the relation between stress and surface density in the alpha model on this branch (see Appendix A). It is possible that these differences may also be relevant to evolution on the inflow time scale in the gas and radiation pressure dominated regimes in real MRI turbulence.

\section{Conclusions}

We have completed vertically stratified, local radiation MHD simuations of magnetorotational turbulence with fixed vertical gravity over a range of surface mass densities. All of the simulations reach a thermal equilibrium, but with continued long term fluctuations in the in-

ternal energy content. We have confirmed earlier work (Turner 2004; Hirose, Krolik, \& Blaes 2009) that the radiation pressure dominated thermal instability predicted by the standard alpha disk model does not exist, even though the the box- and time-averaged radiation to gas pressure ratios in the new simulations are as high as 70.

However, we also find that, when averaged over many thermal times, the vertically integrated total thermal pressure (i.e., radiation plus gas pressure) is well-correlated with the vertically integrated stress. Neither the vertically integrated gas pressure nor the geometric mean of gas and radiation pressure exhibit such a good correlation. The same simulation data therefore yield a thermal equilibrium relation between surface density and (long) timeaveraged vertically integrated stress that follows the one predicted by the traditional alpha model: $\left\langle W_{r \phi}\right\rangle \propto \Sigma^{-1}$. Consequently, if thermal equilibrium is maintained on the long time scales associated with radial mass transport by the turbulent stresses, these local results 
suggest that the nonlocal clumping of mass associated with the classic Lightman \& Eardley (1974) inflow instability might develop. Radiation MHD shearing box simulations with much wider boxes in the radial direction, or well-resolved global simulations, will be necessary to investigate this possibility.

This work was partially supported by a Grant-in-Aid for Scientific Research (No. 20340040) from the Ministry of Education, Culture, Sports, Science and Technology of Japan; and by NSF grants AST-0707624 and AST-0507455. Numerical computations were carried out partially on the Cray XT4 at the Center for Computational Astrophysics at the National Astronomical Observatory of Japan, and on the SX8 at the Yukawa Institute for Theoretical Physics at Kyoto University. We thank Steve Balbus, Shane Davis, Chris Done, Jeremy Goodman, John Hawley, Phil Marshall, Gordon Ogilvie, John Papaloizou, Eli Rykoff, Chris Reynolds, and Jim Stone for very useful conversations. We also thank the anonymous referee for helpful comments that improved the manuscript.

\section{A. The Stress/Surface Density Relation for Alpha-Disks Accounting for Internal Structure from the Simulations}

We derive one-zone vertical structure equations for an alpha-disk in terms of parameters measured from the simulations as follows. Vertical hydrostatic equilibrium implies that the midplane pressure is given by

$$
P(0)=\frac{1}{2} \Omega^{2} \Sigma H_{\rho 1},
$$

where $H_{\rho 1}$ is a density scale height defined through the first vertical moment of the density distribution,

$$
H_{\rho 1} \equiv \frac{2}{\Sigma} \int_{0}^{\infty} \rho(z) z d z
$$

The midplane pressure in the simulations is always dominated by gas and radiation pressure,

$$
P(0)=\frac{1}{3} a T(0)^{4}+\frac{\Sigma k T(0)}{2 \mu H_{\rho 0}},
$$

where $T(0)$ is the midplane temperature and $\mu=0.6$ atomic mass units is the mean particle mass assumed in the simulations. We have eliminated the midplane density by defining the zeroth vertical moment of the density distribution,

$$
H_{\rho 0} \equiv \frac{\Sigma}{2 \rho(0)} \equiv \frac{1}{2 \rho(0)} \int_{-\infty}^{\infty} \rho(z) d z
$$

The fundamental assumption of an alpha-disk model is that the vertically integrated stress $W_{r \phi}$ is $\alpha$ times the vertical integral of the thermal pressure $P_{\text {th }}$. We may therefore 
write

$$
W_{r \phi}=2 \alpha H_{P} P(0)
$$

where the thermal pressure scale height has been defined as

$$
H_{P} \equiv \frac{1}{2 P(0)} \int_{-\infty}^{\infty} P_{\mathrm{th}}(z) d z
$$

Thermal equilibrium implies that the flux $F=\sigma T_{\text {eff }}^{4}$ emerging from each face of the disk is given by

$$
F=-\frac{1}{2} W_{r \phi} r \frac{d \Omega}{d r}=\frac{3}{4} \Omega W_{r \phi}
$$

Finally, the fact that most of the accretion power ultimately escapes vertically through radiative diffusion in the simulations motivates us to write the emergent flux as

$$
F=\xi \frac{2 a c T(0)^{4}}{3 \kappa \Sigma}
$$

where the opacity is dominated by electron scattering in our simulations, $\kappa \simeq 0.33$. We have introduced a parameter $\xi$ which is usually taken to be approximately unity in alphadisk models. As we discuss below, however, our measured values of $\xi$ in the simulation are substantially greater than unity. This is presumably due to the fact that the dissipation profile peaks off the midplane, and that a non-negligible fraction of the accretion power is transported away from the midplane by mechanical motions.

Equations (A1), (A3), (A5), (A7), and (A8) can be combined to give the relationship between emergent flux or vertically integrated stress and surface density. In the gas and radiation pressure dominated limits, the result is

$$
F=\frac{3}{4} \Omega W_{r \phi}= \begin{cases}{\left[\frac{3^{5} \kappa}{2^{9} a c \xi}\left(\frac{\alpha \Omega k}{\mu}\right)^{4}\left(\frac{H_{P}}{H_{\rho 0}}\right)^{4}\right]^{1 / 3} \Sigma^{5 / 3},} & \text { if } P_{\text {gas }}(0) \gg P_{\text {rad }}(0) \\ \frac{4 c^{2} \Omega}{3 \alpha \kappa^{2}} \xi^{2}\left(\frac{H_{\rho 1}}{H_{P}}\right) \Sigma^{-1}, & \text { if } P_{\text {rad }}(0) \gg P_{\text {gas }}(0) .\end{cases}
$$

This gives the usual result that the alpha disk is inflow stable if gas pressure dominated, but inflow unstable if radiation pressure dominated. Note that the hydrostatic equilibrium equation (A1) is not needed to derive the gas pressure dominated relation.

If neither pressure dominates at the midplane, the following equation can be used to derive the relationship between flux or stress and surface density:

$$
5 \tilde{F}^{3 / 4}-2 \tilde{\Sigma}^{5 / 4}-3 \tilde{F}^{5 / 4} \tilde{\Sigma}^{1 / 2}=0
$$


where $\tilde{\Sigma} \equiv \Sigma / \Sigma_{\text {crit }}, \tilde{F} \equiv F / F_{\text {crit }}$,

$$
\Sigma_{\text {crit }}=\left[\frac{2^{19}}{(9) 5^{10}} \frac{a}{\Omega}\left(\frac{c \xi}{\alpha \kappa}\right)^{7}\left(\frac{\mu}{k}\right)^{4} \frac{H_{\rho 1}^{3} H_{\rho 0}^{4}}{H_{P}^{7}}\right]^{1 / 8}
$$

and

$$
F_{\text {crit }}=\frac{12 c^{2} \xi^{2} \Omega}{25 \alpha \kappa^{2} \Sigma_{\text {crit }}}\left(\frac{H_{\rho 1}}{H_{P}}\right) .
$$

Apart from the extra dimensionless factors of $\xi$ and the pressure and density scale heights, equation (A11) agrees with the expression for the maximum surface density consistent with thermal equilibrium that was first derived by Lightman (1974a).

Equations (A9) and (A10) are what we use to plot the alpha disk predictions in Figure 4. We measured the dimensionless parameters describing the internal structure of the disk as follows. Using the time series of horizontally-averaged data from each simulation, we measured $\alpha$ as the ratio of the vertically integrated stress to vertically-averaged pressure, and $\xi$ in terms of the ratio of emerging flux (averaged over both faces of the disk) to midplane radiation energy density. We then averaged both of these quantities over time. We measured the scale height ratios $H_{\rho 0} / H_{\mathrm{P}}$ and $H_{\rho 1} / H_{\mathrm{P}}$ from the time and horizontally-averaged vertical profiles of density and thermal pressure from each simulation. The results are shown in Table 2 .

The scale height ratio parameters are remarkably constant across all the simulations. The numerical values of these parameters are close to what one would obtain from simple analytic equilibria in a gravitational field that increases linearly with height. An $n=3$ polytrope (adiabatic, radiation pressure dominated) has $H_{\rho 0} / H_{P}=1.125$ and $H_{\rho 1} / H_{P} \simeq$ 0.67, while an $n=3 / 2$ polytrope (adiabatic, gas pressure dominated) has $H_{\rho 0} / H_{P}=1.2$ and $H_{\rho 1} / H_{P} \simeq 0.69$. Note that both ratios are slightly larger in the gas pressure dominated polytrope, in agreement with the trend that we see in the simulations. These simple models do not agree exactly with the simulations due in part to nonzero entropy gradients. In the simulations, the entropy generally increases away from the midplane, so that the density must decrease faster for a given pressure decrease compared to an adiabatic profile. Hence the ratio of density scale height to pressure scale height is smaller than that of an adiabatic profile, and this is why the ratios we measure in the simulations are slightly smaller than the polytropic ratios.

In contrast to the scale height ratios, the stress parameter $\alpha$ shows a little more scatter. The parameter $\xi$ clearly increases as the simulations become more radiation pressure dominated. 


\section{B. The Radial Mass Diffusion Equation for the Shearing Box}

The radial mass diffusion equation can be derived from the equations of the shearing

box (Hawley, Gammie, \& Balbus 1995) as follows. Define the azimuthal averaged surface density at some radius $x$ and time $t$ as

$$
\Sigma(x, t) \equiv \frac{1}{L_{y} \Delta x} \int_{x-\Delta x / 2}^{x+\Delta x / 2} d x^{\prime} \int_{-L_{y} / 2}^{L_{y} / 2} d y \int_{-L_{z} / 2}^{L_{z} / 2} d z \rho\left(x^{\prime}, y, z, t\right)
$$

and the mass-weighted vertical and azimuthal average of some quantity $X$ as

$$
<X>(x, t)=\frac{1}{L_{y} \Delta x \Sigma} \int_{x-\Delta x / 2}^{x+\Delta x / 2} d x^{\prime} \int_{-L_{y} / 2}^{L_{y} / 2} d y \int_{-L_{z} / 2}^{L_{z} / 2} d z \rho\left(x^{\prime}, y, z, t\right) X\left(x^{\prime}, y, z, t\right) .
$$

Here we have also performed a radial average over a length scale $\Delta x$ of order an assumed radial coherence length of the turbulence, which is presumably of order the disk scale height. Apart from the use of Cartesian coordinates in a box, these definitions are exactly the same as the vertical integrations and averages used to derive the alpha-disk equations from the MHD equations by Balbus \& Papaloizou (1999). Applying this averaging and vertical integration to the mass continuity and $y$-momentum equations of the shearing box, we obtain

$$
\frac{\partial \Sigma}{\partial t}+\frac{\partial}{\partial x}\left(\Sigma<v_{x}>\right)=0
$$

and

$$
\frac{\partial}{\partial t}\left(\Sigma<v_{y}>\right)+\frac{\partial}{\partial x}\left(\Sigma<v_{x}><v_{y}>\right)+\frac{\partial W_{x y}}{\partial x}=-2 \Omega \Sigma<v_{x}>,
$$

where $W_{x y}$ is the vertically-integrated and azimuthally-averaged Maxwell and Reynolds stress,

$$
W_{x y}(x, t)=\frac{1}{L_{y}} \int_{-L_{y} / 2}^{L_{y} / 2} d y \int_{-L_{z} / 2}^{L_{z} / 2} d z\left(-\frac{B_{x} B_{y}}{4 \pi}+\rho v_{x} \delta v_{y}\right) .
$$

Radial derivatives are defined through differences over the length scale $\Delta x$. We have also assumed zero mass and Poynting flux through the vertical boundaries, and $\left\langle\delta v_{y}\right\rangle=0$. Equations (B33) and ( $(\underline{B} 4)$ can then be combined into a radial mass diffusion equation,

$$
\frac{\partial \Sigma}{\partial t}=\frac{1}{(2-q) \Omega} \frac{\partial^{2}}{\partial x^{2}} W_{x y}
$$

where $q \equiv-d \ln \Omega / d \ln r$ is the shear parameter. 


\section{REFERENCES}

Balbus, S. A., \& Hawley, J. F. 1998, Rev. Mod. Phys., 70, 1

Balbus, S. A., \& Papaloizou, J. C. B. 1999, ApJ, 521, 650

Belloni, T., Méndez, M., King, A. R., van der Klis, M., \& van Paradijs, J. 1997, ApJ, 479, L145

Blaes, O., Hirose, S., \& Krolik, J. H. 2007, ApJ, 664, 1057

Burm, H. 1985, A\&A, 143, 389

Done, C., Wardziński, G., \& Gierliński, M. 2004, MNRAS, 349, 393

Hawley, J. F., Gammie, C. F., \& Balbus, S. A. 1995, ApJ, 440, 742

Hawley, J.F. \& Krolik, J.H. 2001, ApJ 548, 348

Hawley, J.F. \& Krolik, J.H. 2002, ApJ 566, 164

Hirose, S., Krolik, J. H., \& Stone, J. M. 2006, ApJ, 640, 901

Hirose, S., Krolik, J. H., \& Blaes, O. 2009, ApJ, 691, 16

Honma, F., Matsumoto, R., \& Kato, S. 1991, PASJ, 43, 147

Krolik, J. H., Hirose, S., \& Blaes, O. 2007, ApJ, 664, 1045

Lasota, J.-P. 2001, New Astron. Rev., 45, 449

Lightman, A. P. 1974a, ApJ, 194, 419

Lightman, A. P. 1974b, ApJ, 194, 429

Lightman, A. P., \& Eardley, D. M. 1974, ApJ, 187, L1

Lynden-Bell, D., \& Pringle, J. E. 1974, MNRAS, 168, 603

Merloni, A. 2003, MNRAS, 341, 1051

Merloni, A., \& Fabian, A. C. 2002, MNRAS, 332, 165

Ohsuga, K., Mineshige, S., Mori, M., \& Kato, Y. 2009, PASJ, in press

Sakimoto, P. J., \& Coroniti, F. V. 1981, ApJ, 247, 19 
Sakimoto, P. J., \& Coroniti, F. V. 1989, ApJ, 342, 49

Shakura, N. I., \& Sunyaev, R. A. 1973, A\&A, 24, 337

Shakura, N. I., \& Sunyaev, R. A. 1976, MNRAS, 175, 613

Shibazaki, N., \& Hōshi, R. 1975, Prog. Theor. Phys., 54, 706

Smak, J. 1984, PASP, 96, 5

Stella, L., \& Rosner, R. 1984, ApJ, 277, 312

Szuszkiewicz, E. 1990, MNRAS, 244, 377

Szuszkiewicz, E., \& Miller, J. C. 1998, MNRAS, 298, 888

Turner, N. J., 2004, ApJ, 605, L45 


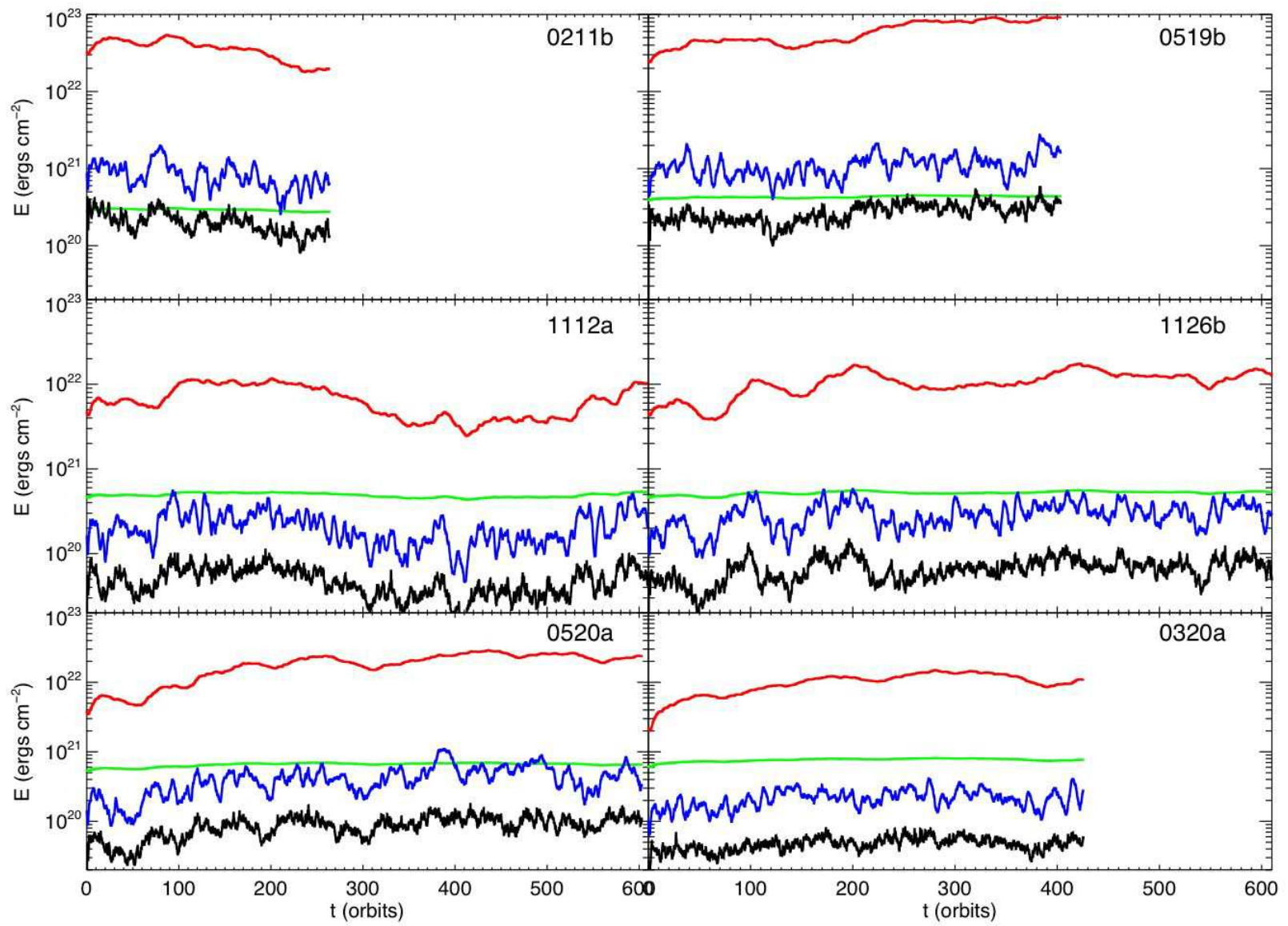

Fig. 1. - Time history of the box integrated radiation internal energy (red), gas internal energy (green), magnetic energy (blue) and turbulent kinetic energy (black) for each of the radiation pressure dominated simulations. 


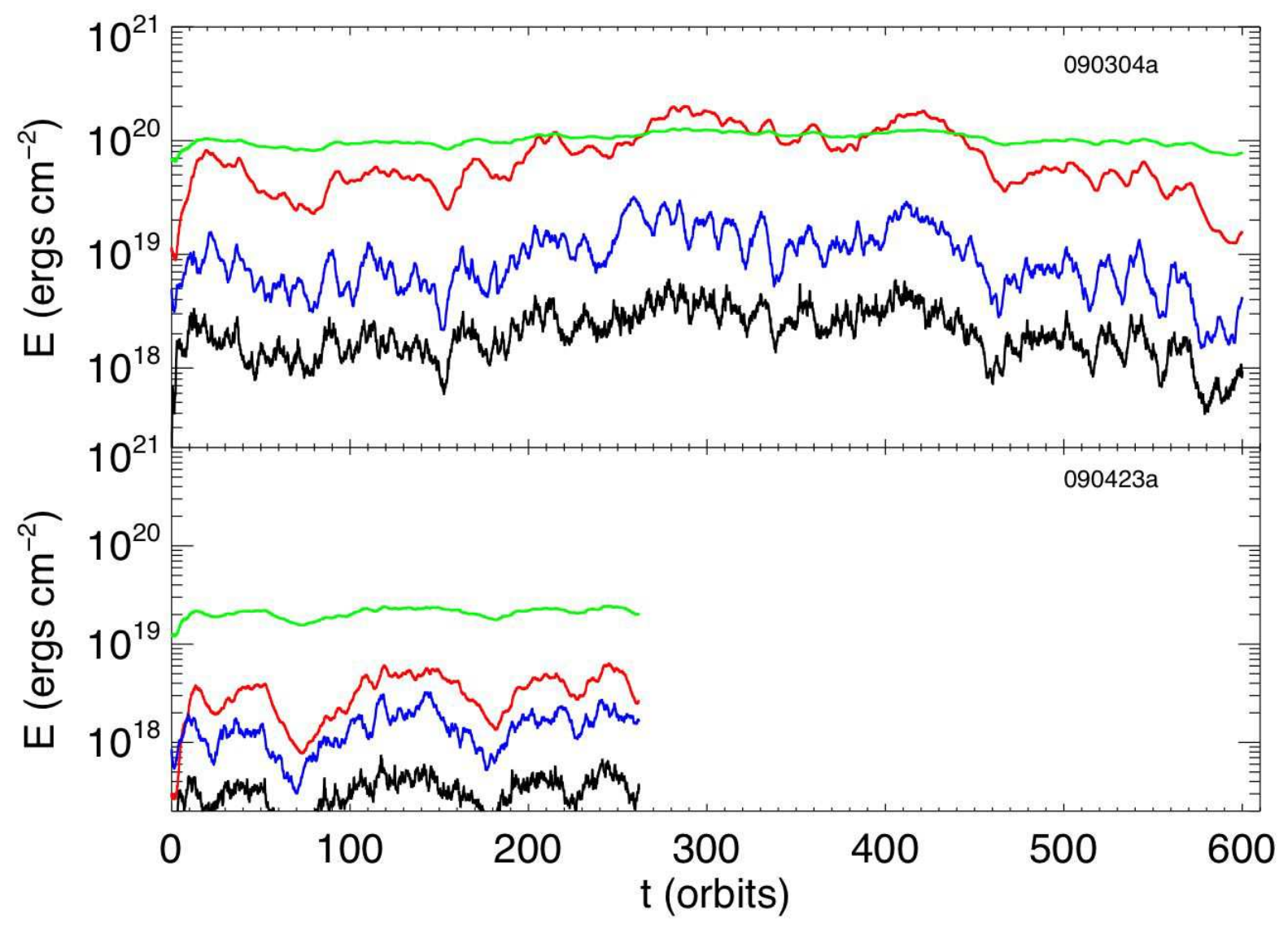

Fig. 2.- Same as Figure 1 except for simulations 090304a and 090423a, which are not radiation pressure dominated. Note the much smaller energies on the vertical axis. 


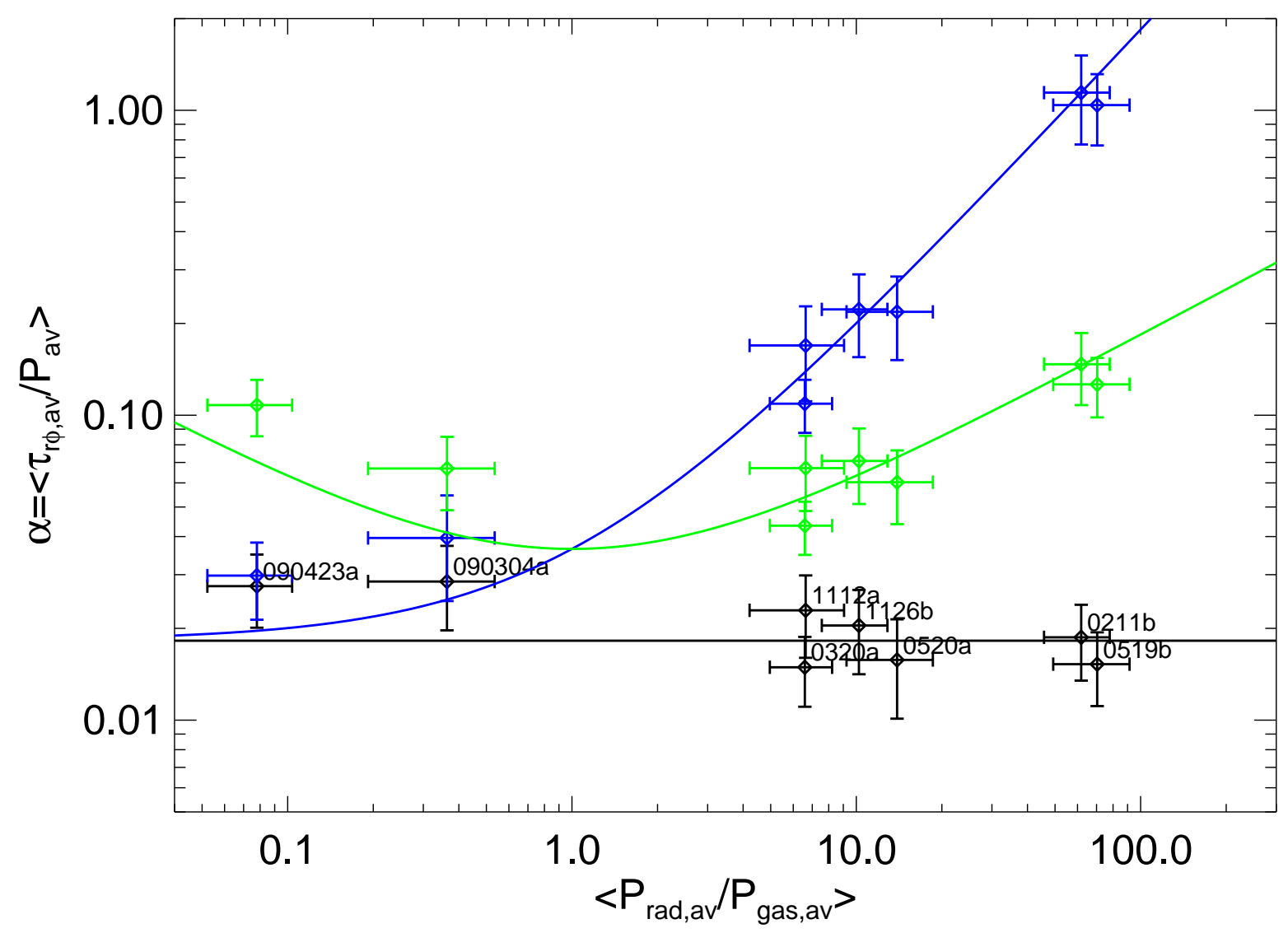

Fig. 3.- Measured values of the stress parameter $\alpha$ as a function of the time-averaged ratio of the box-averaged radiation pressure to the box-averaged gas pressure. The black points define $\alpha$ as the time-averaged ratio of the vertically averaged stress to the box-averaged total thermal pressure. The blue and green points define $\alpha$ in the same way except with total thermal pressure replaced by gas pressure and the geometric mean of gas and radiation pressure, respectively. Both horizontal and vertical error bars indicate one standard deviation in the time averages. The horizontal black line indicates the weighted mean of $\alpha$ for the total pressure stress prescription. Assuming that stress really scales with total thermal pressure, the green and blue curves show how $\alpha$ would then behave under the other stress prescriptions. 


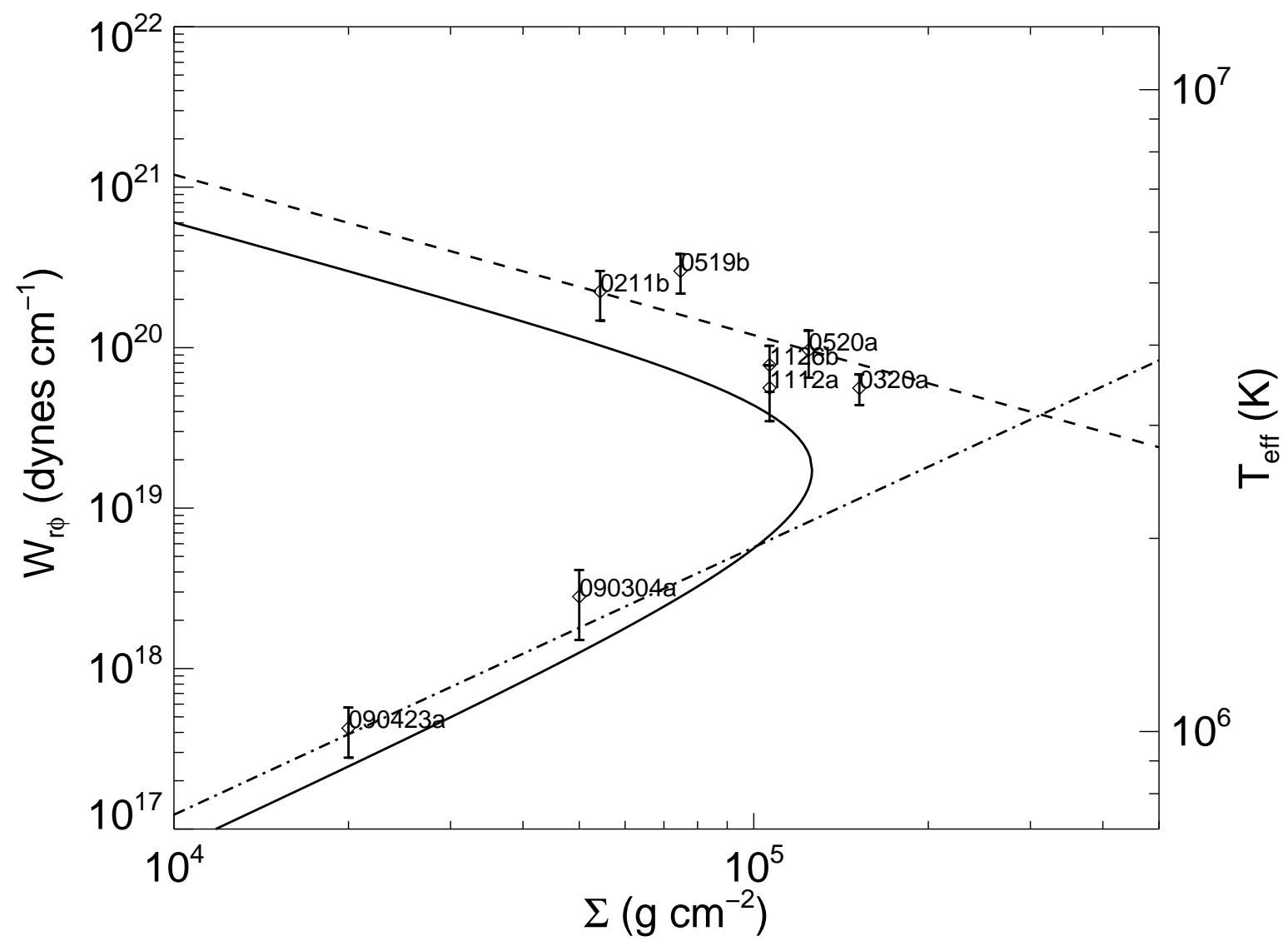

Fig. 4.- Time averaged, vertically integrated stress as a function of surface mass density for each simulation. The right hand axis shows the corresponding effective temperature of the radiation leaving each vertical face of the box. The solid curve shows the prediction of the vertically integrated alpha disk model with internal structure parameters averaged over all the simulations. The dashed line shows the prediction of the radiation pressure dominated alpha disk model with internal structure parameters averaged over the radiation pressure dominated simulations. The dot-dashed line shows the prediction of the gas pressure dominated alpha disk model with internal structure parameters measured from the gas pressure dominated simulations. (See Appendix A for the equations used to define the internal structure parameters.) 
Table 1. Simulation Parameters

\begin{tabular}{cccccc}
\hline \hline Simulation & $\begin{array}{c}\Sigma \\
\left(\mathrm{g} \mathrm{cm}^{-2}\right)\end{array}$ & $\begin{array}{c}H \\
(\mathrm{~cm})\end{array}$ & $\begin{array}{c}\text { Duration } \\
(\text { orbits })\end{array}$ & $\begin{array}{c}\text { Box Dimensions } \\
\left(L_{x} / H \times L_{y} / H \times L_{z} / H\right)\end{array}$ & $\begin{array}{c}\text { Grid Zones } \\
\left(N_{x} \times N_{y} \times N_{z}\right)\end{array}$ \\
\hline $0211 \mathrm{~b}$ & $5.43 \times 10^{4}$ & $5.83 \times 10^{6}$ & 264 & $0.3375 \times 1.35 \times 6.3$ & $48 \times 96 \times 896$ \\
$0519 \mathrm{~b}$ & $7.48 \times 10^{4}$ & $4.37 \times 10^{6}$ & 403 & $0.3375 \times 1.35 \times 6.3$ & $48 \times 96 \times 896$ \\
$1112 \mathrm{a}$ & $1.06 \times 10^{5}$ & $1.46 \times 10^{6}$ & 610 & $0.45 \times 1.8 \times 8.4$ & $48 \times 96 \times 896$ \\
$1126 \mathrm{~b}$ & $1.06 \times 10^{5}$ & $1.46 \times 10^{6}$ & 611 & $0.45 \times 1.8 \times 8.4$ & $48 \times 96 \times 896$ \\
$0520 \mathrm{a}$ & $1.24 \times 10^{5}$ & $1.17 \times 10^{6}$ & 603 & $0.54 \times 2.16 \times 10.08$ & $48 \times 96 \times 896$ \\
$0320 \mathrm{a}$ & $1.52 \times 10^{5}$ & $7.28 \times 10^{5}$ & 426 & $0.6 \times 2.4 \times 11.2$ & $48 \times 96 \times 896$ \\
$090304 \mathrm{a}$ & $5.00 \times 10^{4}$ & $3.13 \times 10^{5}$ & 600 & $0.625 \times 2.5 \times 10$ & $32 \times 64 \times 512$ \\
$090423 \mathrm{a}$ & $2.00 \times 10^{4}$ & $2.10 \times 10^{5}$ & 262 & $0.5 \times 2.0 \times 8.0$ & $32 \times 64 \times 512$ \\
\hline
\end{tabular}

Table 2. Internal Structure Parameters Measured from Simulations

\begin{tabular}{cccccc}
\hline \hline Simulation & $\begin{array}{c}\Sigma \\
\left(\mathrm{g} \mathrm{cm}^{-2}\right)\end{array}$ & \multicolumn{1}{c}{$\alpha$} & $\xi$ & $H_{\rho 0} / H_{\mathrm{P}}$ & $H_{\rho 1} / H_{\mathrm{P}}$ \\
\hline $0211 \mathrm{~b}$ & $5.43 \times 10^{4}$ & $0.019 \pm 0.005$ & $5.4 \pm 1.2$ & 0.952 & 0.629 \\
$0519 \mathrm{~b}$ & $7.48 \times 10^{4}$ & $0.015 \pm 0.004$ & $5.6 \pm 1.3$ & 0.949 & 0.630 \\
$1112 \mathrm{a}$ & $1.06 \times 10^{5}$ & $0.023 \pm 0.007$ & $4.5 \pm 1.1$ & 0.934 & 0.624 \\
$1126 \mathrm{~b}$ & $1.06 \times 10^{5}$ & $0.020 \pm 0.006$ & $4.7 \pm 1.0$ & 0.948 & 0.632 \\
$0520 \mathrm{a}$ & $1.24 \times 10^{5}$ & $0.016 \pm 0.006$ & $4.6 \pm 1.0$ & 0.926 & 0.629 \\
$0320 \mathrm{a}$ & $1.52 \times 10^{5}$ & $0.015 \pm 0.004$ & $4.1 \pm 0.8$ & 0.990 & 0.645 \\
$090304 \mathrm{a}$ & $5.00 \times 10^{4}$ & $0.028 \pm 0.009$ & $2.8 \pm 0.6$ & 1.090 & 0.680 \\
$090423 \mathrm{a}$ & $2.00 \times 10^{4}$ & $0.028 \pm 0.007$ & $2.3 \pm 0.4$ & 1.127 & 0.685 \\
\hline Mean $_{\text {rad }}{ }^{\mathrm{a}}$ & & $0.017 \pm 0.002$ & $4.7 \pm 0.4$ & 0.950 & 0.631 \\
Mean $_{\text {gas }} \mathrm{b}$ & & $0.028 \pm 0.006$ & $2.5 \pm 0.4$ & 1.109 & 0.683 \\
Mean $_{\text {all }}{ }^{\mathrm{c}}$ & & $0.018 \pm 0.002$ & $3.4 \pm 0.3$ & 0.990 & 0.644 \\
\hline
\end{tabular}

${ }^{a}$ Averaged over the six radiation pressure dominated simulations, i.e. excluding 090304a and 090423a.

${ }^{\mathrm{b}}$ Averaged over the the gas pressure dominated simulations 090304a and 090423a.

${ }^{\mathrm{c}}$ Averaged over all eight simulations. 